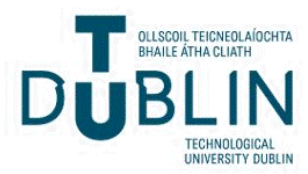

Technological University Dublin ARROW@TU Dublin

\section{We can't afford to turn a blind eye to myopia}

\author{
Christine Marie Bourke \\ The Mater Misericordiae University Hospital \\ James Loughman \\ Technological University Dublin, james.loughman@tudublin.ie \\ Ian Flitcroft \\ Children's University Hospital, Temple Street, Dublin, Ireland
}

See next page for additional authors

Follow this and additional works at: https://arrow.tudublin.ie/scschphyart

Part of the Optometry Commons

\section{Recommended Citation}

Bourke CM, Loughman J, Fltcroft DI, Loskutova E, O'Brien C. We can't afford to turn a blind eye to myopia, QJM: An International Journal of Medicine, 2019;hcz076, https://doi.org/10.1093/qjmed/hcz076

This Article is brought to you for free and open access by the School of Physics \& Clinical \& Optometric Science at ARROW@TU Dublin. It has been accepted for inclusion in Articles by an authorized administrator of ARROW@TU Dublin. For more information, please contact arrow.admin@tudublin.ie, aisling.coyne@tudublin.ie, gerard.connolly@tudublin.ie.

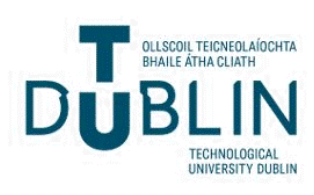


Authors

Christine Marie Bourke, James Loughman, Ian Flitcroft, Ekaterina Loskutova, and Colm O’Brien 


\title{
We can't afford to turn a blind eye to myopia
}

Authors: Dr Christine Mary Bourke, The Mater Misericordiae University Hospital, Eccles Street, Inns Quay, Dublin, D07R2WY; Childrens University Hospital Temple Street, Temple St, Dublin 1, D01YC67. (First Author)

Professor James Loughman, Centre for Eye Research Ireland, Dublin Institute of Technology City Campus, Dublin, D07HK68

Professor Daniel Ian Fltcroft, Childrens University Hospital Temple Street, Temple Street, Dublin 1, D 01YC67.

Ms Ekatherina Loskutova, Centre for Eye Research Ireland, Dublin Institute of Technology City Campus, Dublin, D07HK68.

Professor Colm O'Brien, The Mater Misericordiae University Hospital, Eccles Street, Inns Quay, Dublin, D07R2WY. (Final Author)

Correponding author: Dr Christine Mary Bourke, Ophthalmology Department, Childrens University Hospital Temple Street, Temple St, Dublin 1, D01YC67.

Telephone: 0857504663

Email: bourkecm@tcd.ie

\begin{abstract}

\section{Background}

Myopia is becoming increasingly prevalent throughout the world. It is an overlooked but leading cause of blindness, particularly among the working aged population. Myopia is often considered benign because it is easily corrected with glasses, contact lenses, or refractive
\end{abstract}


surgery. Traditionally myopia has been classified into physiological and pathological subtypes based on the degree of myopia present. Higher levels of myopia are associated with increased risk of pathological complications but it is important to note that there is no safe level of myopia. Even low levels of myopia increase the risk of retinal detachment and other ocular comorbidities which will be discussed in detail later. The most serious complication, myopic maculopathy, is the only leading cause of blindness without an established treatment and therefore leads to inevitable loss of vision in some myopes, even at a young age.

Aim: To highlight the current myopia epidemic and the sight threatening complications associated with it

Design: This is a comissioned review article. Data was gathered by performing a literature review, searching the PubMed database for recent articles regarding myopia.

Conclusions: Myopia is a potentially blinding disease. By identifying at risk individuals and intervening before they become myopic, eye care practitioners can prevent or delay spectacle use, reduce the risk of the myriad of myopic complications and thereby improve the patient's quality of life and positively impact its socio-economic effects.

\section{Main Article}

\section{Introduction}

The concept of myopia originated with Aristotle (350 BC), who used the word $\mu \cup \dot{\omega} \psi \varsigma$ (muoops) derivated from $\mu$ úcı (muein, to close) and $\omega \psi$ (oops, the eye). ${ }^{1}$ Around 1700 , 
increased ocular length was noted to be associated with myopia. ${ }^{2}$ Theories about the condition continued to be developed throughout the 1800's and beyond. ${ }^{3}$ Myopia or nearsightedness occurs when parallel rays of light are focused in front of the retina rendering the patient unable to see in the distance. It can be defined, from an optical perspective, as a refractive anomaly of the non-accommodated eye which can be corrected using concave (negatively powered) lenses. It can also be defined physiologically, as an abnormality of eye growth resulting in excessive axial elongation of the eye. ${ }^{4}$

Why is myopia important? Firstly, the condition is becoming increasingly prevalent throughout the world; secondly, it is an overlooked but leading cause of blindness, particularly among the working aged population. Myopia is often considered benign because it is easily corrected with glasses, contact lenses, or refractive surgery. Traditionally myopia has been classified into physiological and pathological subtypes based on the degree of myopia present. Higher levels of myopia are associated with increased risk of pathological complications but it is important to note that there is no safe level of myopia. Even low levels of myopia increase the risk of retinal detachment and other ocular comorbidities which will be discussed in detail later. The most serious complication, myopic maculopathy, is the only leading cause of blindness without an established treatment and therefore leads to inevitable loss of vision in some myopes, even at a young age.

\section{Prevalence}

Myopia is the most common ocular abnormality in the world. ${ }^{5,6}$ With an estimated 1.406 billion people with myopia (22.9\% of the world population) and 163 million people with high myopia ( $2.7 \%$ of the world population) in the year 2000 , the disorder has significant public healthcare implications worldwide, and poses a significant societal and economic burden to healthcare systems globally. ${ }^{4}$ Current estimates predict that myopia and high myopia will significantly increase, to affect nearly 5 billion ( $50 \%$ of the projected global population), and 1 billion respectively, in $2050 .^{6}$

Rates as high as $96.5 \%$ have been reported in young males in South Korea. ${ }^{7}$ Its prevalence is increasing, not only in East Asian populations, but also in the West. ${ }^{8,9}$ Findings from the Northern Ireland Childhood Errors of Refraction (NICER) study show that nearly one in five teenagers in the UK are myopic, with myopia being more than twice as prevalent among UK children now than in the 1960 's (16.4\% vs $7.2 \%) .{ }^{10}$ Furthermore over $50 \%$ of British University students are myopic. ${ }^{11}$ The figures for the Republic of Ireland are similar where the prevalence of myopia in children aged $12-13$ years is $19.9 \%$. ${ }^{12}$ Within the United States myopia prevalence has doubled in just 30 years and now affects almost 1 in 2 people there. Furthermore, vision impairment in the US is projected to increase by $26 \%$ by 2060 , with almost $70 \%$ of cases caused by refractive error. ${ }^{13}$ Hence, the increasing prevalence of myopia isn't solely an Asian problem; it is very much relevant as a global public health concern. 


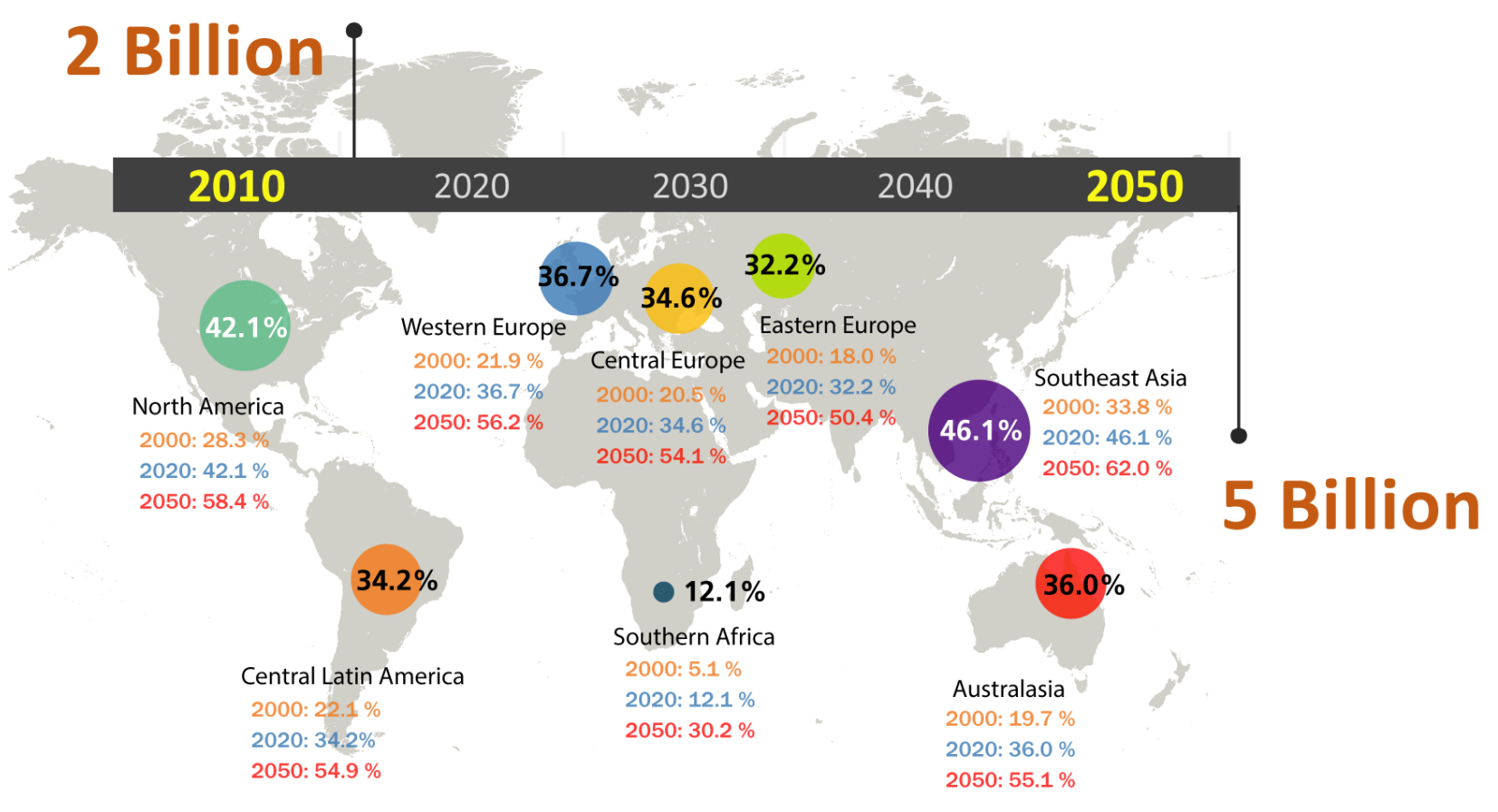

Figure 1. Regional prevalence of myopia and projected trends from 2000 through 2050 (Data sourced from Holden et al.) ${ }^{6}$

\section{Risk factors for myopia development}

Myopia is likely due to a combination of environmental and genetic factors that influence the normal growth mechanisms of the eye. Genetic factors are well established as a cause of myopia. Although the specific genes and pathways are not yet fully defined, several genes have been identified that are associated with high myopia. There is also good evidence for gene-environment interactions. A recent study demonstrated that the impact of a specific gene (APLP2) on myopia was dependent on reading behaviour.${ }^{14}$

Analysis of syndromic forms of myopia has also recently provided novel insights into the genetics of myopia, with 21 novel candidate genes and genetic pathways identified, thereby providing further insights into the aetiology of myopia. ${ }^{15}$ Children with one myopic parent are almost three times more likely to be myopic by age 13 than a child without a myopic parent. This increases to over seven times more likely when both parents are myopic. ${ }^{10}$

It is now widely accepted, however, that environmental, lifestyle and behavioural factors are driving the current change in prevalence. Many epidemiological surveys have shown that excessive close-up work, a high level of education, and reduced participation in outdoor activities are important environmental risk factors for myopia. ${ }^{16,17} \mathrm{~A}$ recent study using the technique of Mendelian Randomisation has provided clear evidence of a causal relationship between increased education and myopia. ${ }^{18}$ 
More time spent outdoors and illiteracy have long been associated with a reduced incidence of myopia. ${ }^{19}$ Although twin studies show an apparent high heritability of refractive errors, the dramatic and precipitous recent changes in prevalence of myopia cannot be explained by changes in the gene pool. ${ }^{20}$ People who are not genetically predisposed, it seems, are becoming myopic due to lifestyle and behavioural choices.

Increasing urbanisation also appears to be a factor. A recently published systematic review examined the global variation of childhood myopia and found that children living in predominantly urban environments have 2.6 times the risk of myopia compared to children living in rural environments. ${ }^{19}$ Potential explanations include a more congested environment, a greater emphasis on education, and as a result more near-vision work and fewer outdoor activities. In a study involving 863 Australian children, children who were myopic only spent an average of 16.3 hours outdoors per week compared to non-myopic children, who spent 21.0 hours outdoors. Children are now growing up in an era of visually demanding technology, this may be a factor as (a) it competes for leisure time that might otherwise be spent outdoors and (b) it involves extended periods of proximal viewing, often at very close proximity to the eyes, thereby contributing to two of the major risk factors for myopia development. Recent data in Ireland (as yet unpublished-abstract accepted for Association for Research in Vision and Ophthalmology conference, April 2019) revealed that myopic students use twice as much smartphone data as non-myopes. This evolving risk factor requires further exploration. ${ }^{21}$

\section{Economic Burden}

As the prevalence of myopia is increasing dramatically, the large socio-economic costs associated with myopia make the disorder a serious public health issue. ${ }^{22}$ The global productivity lost due to vision impairment caused by uncorrected refractive error is estimated to be $\$ 202$ billion annually. ${ }^{23}$ There are various other costs not included in these calculations, including the cost to government and affected individuals of treating myopia itself, the cost of treating the associated ocular co-morbidities and of managing vision impairment and blindness among those affected. The quality of life impact also needs to be considered in order to get a better indication of the true socio-economic impact of myopia. The public health and economic impact of myopia is extensive and comparable with other major chronic diseases, but crucially, given the nature of the causal mechanisms driving the epidemic, the impact is modifiable with suitable intervention. Thus, myopia warrants significantly more international attention as a global public health concern.

\section{Ocular comorbidities}

In addition to the costs of optical correction, high myopia is associated with significant ocular morbidity and visual disability. While glasses, contact lenses and refractive surgery can effectively address the symptoms of refractive error, myopic patients are at an 
increased risk of numerous secondary sequelae including glaucoma, cataract, optic neuropathy, staphlyoma, retinal detachment and myopic macular degeneration. 24, 25,26

The aforementioned conditions are potentially blinding. The impact of the rise in myopia prevalence is compounded by the fact that the prevalence rises are accompanied by an increase in the average magnitude of myopia, with children becoming myopic at a younger age and progressing faster to higher levels. As the level of risk is associated with the degree of myopia, this places those affected at higher risk of complications and vision loss. Already, in the working population in the UK, Ireland and Europe, myopia is among the leading causes of blindness and becoming a more significant concern. ${ }^{27}$

Myopia represents a major risk factor for ocular disease that is comparable, even for low to moderate levels of myopia, with the risks associated with hypertension for cardiovascular disease. ${ }^{20}$ The myopia risks for glaucoma and cataract are also comparable with the risks of stroke from smoking $>20$ cigarettes per day. For retinal detachment and myopic maculopathy, myopia carries a risk far in excess of any identified population risk factor for cardiovascular disease.

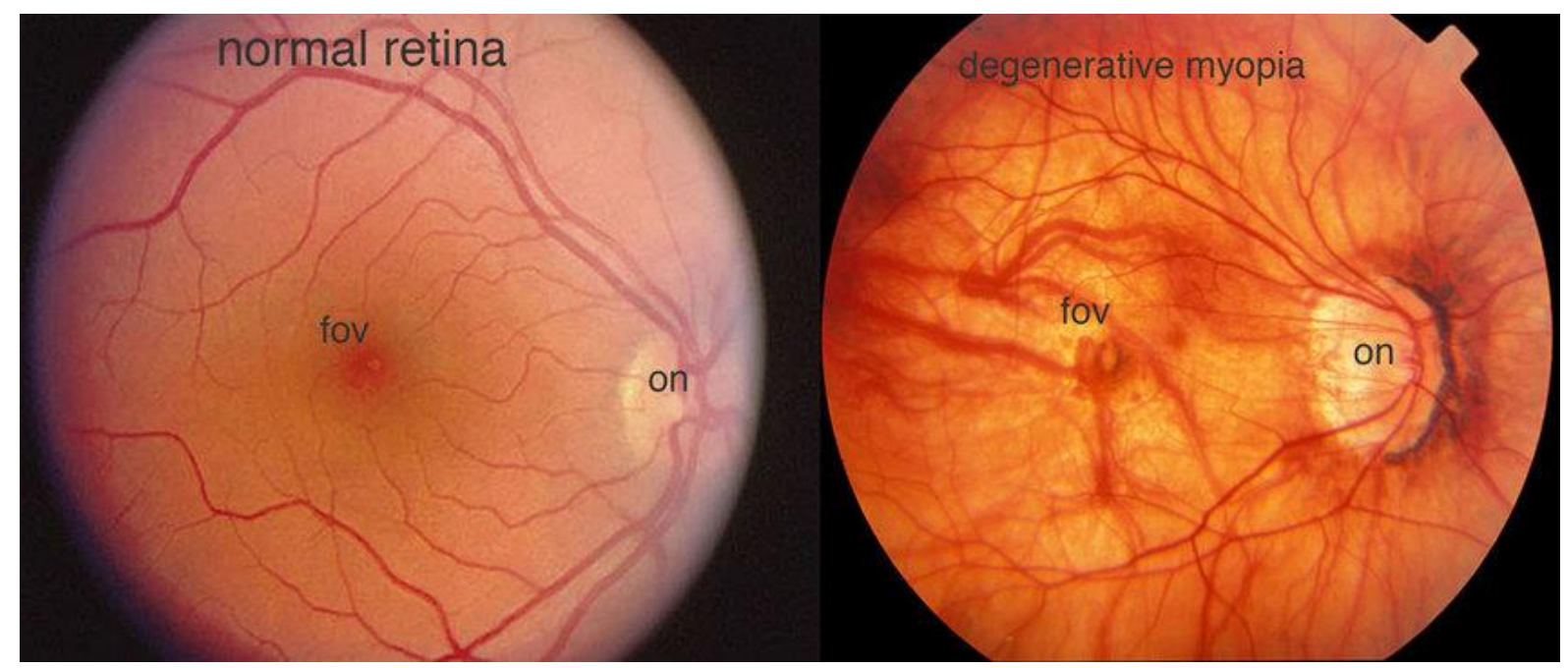

Figure 1: Normal retina in contrast to a retina exhibiting characteristic signs of degenerative myopia. Image : Dr James Gilman and The Moran Eye Centre ophthalmology photography department.

\section{Treatment/prevention/slowing progression}

Efforts to reduce the prevalence and progression of myopia could have profound public health impact. The focus of intervention in the past has been to correct the refractive error, this focus should move towards understanding the underlying pathology and developing strategies to (a) prevent the condition developing in the first instance and (b) control the rate of progression of myopia once it does develop. Several strategies have been developed as a means to try to control myopia progression including the following: (i) optical interventions such as multifocal contact lenses, specialised spectacle lenses and 
orthokeratology; (ii) pharmaceutical interventions such as low dose atropine; (iii) lifestyle interventions such as increased outdoor activity. ${ }^{28}$

Ortho-K lenses worn at night flatten the central cornea by forcing the epithelial cells to move peripherally, resulting in steepening of the peripheral cornea. This reduces peripheral hyperopia, a stimulus for increasing the length of the eye and thereby ameliorates the progression of myopia. Multifocal soft contact lenses similarly impact peripheral hyperopia and exert a similar controlling effect.

Another method that has proven successful involves the use of a muscarinic antagonist eyedrop such as atropine sulphate to slow the rate of eye growth. A recent meta-analysis examining the effectiveness of the different interventions found that the most effective intervention was atropine. ${ }^{28}$ The Myopia Outcomes Study of Atropine In Children (MOSAIC) study currently underway in Ireland is using low dose atropine $(0.01 \%)$ to slow myopia progression, while a similar study is also set to commence in the UK (CHAMP-UK). The investigation of this low concentration of atropine is particularly important as there is evidence to suggest it can slow myopia progression without the visual side effects caused by the higher concentrations currently licensed in the UK and Ireland for ophthalmic use. ${ }^{29}$

Time spent outdoors also seems to be protective as evidenced by studies from Singapore and Australia. ${ }^{30,31}$ There are also studies from Taiwan showing reduced incidence of myopia among children undertaking an intervention called 'recess outside the classroom' whereby children have increased outdoors exposure. This programme lead to a reduced incidence of new myopia in those involved compared to controls. ${ }^{32}$ The precise mechanism of the effect has yet to be elucidated. It has been postulated from animal studies that an increased level of light outdoors may have the effect of increasing retinal dopamine production and release, altering gene expression in the retina, which in turn reduces axial elongation. ${ }^{33}$ There may also be a more straightforward mechanistic explanation involving the increased viewing distance and less visually cluttered environment experienced when outdoors. Encouraging children to spend more time outdoors is simple, inexpensive and likely to benefit their health in general including reducing weight.

\section{The future}

We now have an understanding of the huge impact environmental factors have on the prevalence of myopia. It is necessary to identify those at high risk based on their genetics, their geographic location and their behaviours and provide timely intervention with existing and emerging treatments. Myopia is a potentially blinding disease. By identifying at risk individuals and intervening before they become myopic, eye care practitioners can prevent or delay spectacle use, reduce the risk of the myriad of myopic complications and thereby improve the patient's quality of life and positively impact its socio-economic effects. The demand for eye services is escalating rapidly. Can society make changes to prevent myopia and associated complications? There are many questions that need to be answered and 
much education to be delivered to key stakeholders so that we can curtail the seemingly inexorable rise in myopia, the most common human eye disorder in the world.

References 
1. Magnus $\mathrm{H}$ The knowledge of visual disturbances among the Greeks and Romans. Graefes Archives of Clinical and Experimental Ophthalmology 1877;23:24-48

2. Glauder GF ; Hermann Boerhavens Abhandlung von Augenkrankheiten und deroselben Kur. Herman Boerhaaven's treatise on eye disesases and their cure. Nürnberg: Endterischen Consorten, 1751:288.)

3. de Jong PTVM; Myopia: its historical contexts; British Journal of Ophthalmology 2018;102:1021-1027.

4. Flitcroft DI, He M, Jonas JB, Jong M, Naidoo K et al; International Myopia Institute - Defining and Classifying Myopia: A Proposed Set of Standards for Clinical and Epidemiologic Studies; Investigative Ophthalmology and Visual Science; Feb 2019 Volume 60, Issue 3

5. Chua J, Wong TY. Myopia-the silent epidemic that should not be ignored. JAMA ophthalmology. 2016;134:1363-1364.

6. Holden BA, Fricke TR, Wilson DA et al. Global prevalence of myopia and high myopia and temporal trends from 2000 through 2050.Ophthalmology. 2016; 123:1036-1042.

7. Jung SK, Lee JH, Kakizaki H, Jee D. Prevalence of myopia and its association with body stature and educational level in 19-year-old male conscripts in Seoul, South Korea. Investigative Ophthalmol Visual Science . 2012; 53: 5579-5583.

8. Foster PJ, Jiang Y. Epidemiology of myopia. Eye. 2014;28:202-208.

9. Williams KM, Bertelsen $G$, Cumberland $P$ et al. Increasing prevalence of myopia in Europe and the impact of education. Ophthalmology. 2015;122(7):1489-1497.

10. McCullough SJ, O'Donoghue L, Saunders KJ (2016). Six Year Refractive Change among White Children and Young Adults: Evidence for Significant Increase in Myopia among White UK Children. PLoS ONE 11(1): e0146332. doi:10.1371/journal.pone.0146332

11. Logan NS, Davies LN, Mallen EA, Gilmartin B. Ametropia and ocular biometry in a U.K. university student population. Optometry Vision Science. 2005 Apr;82(4):261-6.

12. Harrington SC, Stack J, Saunders K, et al Refractive error and visual impairment in Ireland schoolchildren British Journal of Ophthalmology Published Online First: 12 October 2018. doi: 10.1136/bjophthalmol-2018-312573

13. Varma R, Tarczy-Hornoch $K$, Jiang X, Visual Impairment in preschool children in the united states: demographic and geographic variations from 2015-2060. JAMA Ophthalmology 2017; 135:610-616.

14. Tkatchenko, AV. Tkatchenko TV,Guggenheim, JA; APLP2 Regulates Refractive Error and Myopia Development in Mice and Humans ; PLoS Genetics. 2015 Aug; 11(8): e1005432.

15. Flitcroft DI, Loughman J, Wildsoet C, et al; Novel Myopia Genes and Pathways Identified From Syndromic Forms of Myopia; Investgative Ophthalmology and Visual Science 2018 Jan; 59(1): 338-348.

16. Guo Y, Liu L, Tang P et al. Outdoor activity and myopia progression in a 4 year fllow up of Chinese primary school children: The Bejing Children Eye Study. PloS One 2017;12:e0175921

17. He M. Xiang F Zeng $Y$ et al. Effect of time spent outdoors at school on the development of myopia among children in China: a randomised clinical trial. JAMA 2015;312:1142-1148

18. Mountjoy E, Davies NM, Plotnikov D, Smith GD, Rodriguez S, Williams CE et al. Education and myopia: assessing the direction of causality by mendelian randomisation; British Medical Journal 2018; $361: k 2022$

19. Rudnicka AR, Kapetanakis VV, Wathern AK, et al; Global variations and time trends in the prevalence of childhood myopia, a systematic review and quantitative meta-analysis: implications for aetiology and early prevention. British Journal of Ophthalmology 2016;100:882-890. 
20. Flitcroft DI; The complex interactions of retinal, optical and environmental factors in myopia aetiology; Progress in Retinal and Eye Research 31 (2012) 622e660

21. Tay SA, Farzavandi S, Tan D. Interventions to Reduce Myopia Progression in Children. Strabismus 2017, 25:1, 23-32, DOI: 10.1080/09273972.2016.1276940

22. Zheng YF, Pan CW, Chay J, Wong TY, Finkelstein E, Saw SM. The economic cost of myopia in adults aged over 40 years in Singapore. Invest Ophthalmol Vis Sci. 2013; 54:7532-7.

23. Fricke TR, Holden BA, Wilson DA et al. Global cost of correcting visual impairment from uncorrected refractive error. Bulletin World Health Organisation. 2012;90:728-738

24. Bobeck S, Modjtahedi MD et al. Public Health Burden and Potential Interventions for Myopia. Ophthalmology. 2018;12:628-630

25. Mitchell P, Hourihan F, Sandbach J, Wang JJ. The relationship between glaucoma and myopia: the Blue Mountains Eye Study. Ophthalmology. 1999 Oct;106(10):2010-5.

26. Kanthan GL, Mitchell P, Rochtchina E, Cumming RG, Wang JJ. Myopia and the long-term incidence of cataract and cataract surgery: the Blue Mountains Eye Study. Clinical and Experimental Ophthalmology. 2014 May-Jun;42(4):347-53. doi: 10.1111/ceo.12206. Epub 2013 Oct 11.

27. Kocur I Resnikoff S; Visual impairment and blindness in Europe and their prevention.British Journal of Ophthalmology 2002 Jul; 86(7): 716-722.

28. Huang J, Wen D, Wang Q, et al. Efficacy comparison of 16 interventions for myopia control in children: a network meta-analysis. Ophthalmology. 2016;123(4):697-708.

29. Loughman, J. Flitcroft ,DI; The acceptability and visual impact of $0.01 \%$ atropine in a Caucasian population; British Journal of Ophthalmology 2016; 100, (11): 1525-1529

30. French AN, Morgan IG, Mitchell P \& Rose KA (2013b): Patterns of myopigenic activities with age, gender and ethnicity in Sydney schoolchildren. Ophthalmic Physiol Opt 33: 318-328.

31. Dharani R, Lee CF, Theng ZX et al. (2012): Comparison of measurements of time outdoors and light levels as risk factors for myopia in young Singapore children. Eye (Lond) 26: 9119183.

32. Cheng CY, Huang W, Su KC, Peng ML, Sun HY \& Cheng HM (2013): Myopization factors affecting urban elementary school students in Taiwan. Optom Vis Sci 90: 400-406. 2

33. Feldkaemper M, Schaeffel F. An updated view on the role of dopamine in myopia. Experimental Eye Research. 2013;114:106-119.

Photograph references

1. source: MR MAGOO PIC https://i.pinimg.com/originals/63/85/1f/63851f5f4bc6d1a2d985ebb5c862d7de.jpg

2. Myopic fundus vs normal fundus Photo by James Gilman and the Moran Eye Centre ophthalmic photo department 